\title{
Influência do método de síntese no processo de pilarização com titânio de uma esmectita da região amazônica
}

\section{(Influence of the synthesis method on the process of pillarization of smectite from the amazonic region with titanium)}

\author{
D. L. Guerra ${ }^{1}$,V. P. Lemos ${ }^{1}$, R. Angélica ${ }^{1}$, C. Airoldi ${ }^{2}$ \\ ${ }^{1}$ Centro de Geociências, Universidade Federal do Pará - UFPA, C.P. 161, Belém, PA 66075-110 \\ ${ }^{2}$ Instituto de Química, Universidade Estadual de Campinas - UNICAMP, C.P. 6154, Campinas, SP 13083-970 \\ dlguerra@ufpa.br
}

\begin{abstract}
Resumo
Amostras de argila esmectítica provenientes do município de Serra Madureira no Estado do Acre foram utilizadas no processo de pilarização. As matrizes natural e pilarizada foram caracterizadas por difração de raios X, espectroscopia no infravermelho com transformada de Fourier, análises térmicas diferencial e gravimétrica, microscopia eletrônica de varredura, espectroscopia de raios X por energia dispersiva e análise textural. As soluções pilarizantes de titânio, $\mathrm{Ti}\left(\mathrm{OC}_{2} \mathrm{H}_{5}\right)_{4}$ e $\mathrm{TiCl}_{4}$, foram analisadas por espectroscopia no infravermelho com transformada de Fourier. O íon intercalante foi obtido através da reação química entre as soluções de $\mathrm{Ti}\left(\mathrm{OC}_{2} \mathrm{H}_{5}\right)_{4}$ e $\mathrm{HCl}$ na proporção aproximada de $\mathrm{HCl} / \mathrm{Ti}=1$ e soluções de $\mathrm{TiCl}_{4}$ em reação com etanol com relação $\mathrm{Ti} /$ argila $=25 \mathrm{mmol} \mathrm{Ti} / \mathrm{g}$. A intercalação da notronita foi efetuada utilizando-se de dois métodos: com a solução de acido clorídrico incorporada paulatinamente na solução de etóxido de titânio, e utilizando o cloreto de titânio em solução de etanol, com vigorosa agitação a $25{ }^{\circ} \mathrm{C}$ durante $3 \mathrm{~h}$ e com calcinação a $450{ }^{\circ} \mathrm{C}$ e $600{ }^{\circ} \mathrm{C}$. Os resultados de difração de raios $\mathrm{X}$ do processo de pilarização mostraram alterações no espaçamento basal de $15,30 \AA$ para $18,76 \AA$, e na área de superfície específica de 44,37 m²/g para 188,72 $\mathrm{m}^{2} / \mathrm{g}$. A estabilidade térmica da argila natural foi otimizada com o processo de pilarização.

Palavras-chave: titânio-argila pilarizada, intercalação, esmectita.
\end{abstract}

Abstract

Smectite clays of the State of Acre, Brazil, were used for the pillarization process. The natural and pillarized matrices were characterized by X-ray diffraction, Fourier transform infrared spectroscopy (FTIR), thermal analysis, scanning electron microscopy, energy dispersive spectroscopy and textural analysis. The titanium pillarized solutions $\mathrm{Ti}\left(\mathrm{OC}_{2} \mathrm{H}_{5}\right)_{4}$ and $\mathrm{TiCl}_{4}$ were analyzed by FTIR. The intercalation ion was obtained by reacting $\mathrm{Ti}\left(\mathrm{OC}_{2} \mathrm{H}_{5}\right)_{4}$ with $\mathrm{HCl}(\mathrm{HCl} / \mathrm{Ti} \sim 1)$ and $\mathrm{TiCl}_{4}$ with ethanol (Ti/clay $\left.\sim 25 \mathrm{mmol} \mathrm{Ti/g}\right)$. The notronite intercalation was carried out using two methods: with dropwise hydrochloric acid in titanium ethoxide solution, and using titanium tetrachloride, kept under vigorous stirring at $25^{\circ} \mathrm{C}$ for $3 \mathrm{~h}$ and calcination at $450{ }^{\circ} \mathrm{C}$ and $600{ }^{\circ} \mathrm{C}$. The X-ray diffraction results of the pillarization process show an increase $(15.30 \AA$ to $18.76 \AA$ ) in the basal spacing of the natural clay and in the specific surface area $\left(44.37 \mathrm{~m}^{2} / \mathrm{g}\right.$ to $\left.188.72 \mathrm{~m}^{2} / \mathrm{g}\right)$ The thermal stability of the natural clay was improved by the pillarization procedure.

Keywords: titanium-pillared clay, intercalation, smectite.

\section{INTRODUÇ̃̃OO}

A pilarização consiste na preparação de agentes pilarizantes (oligomerização) e da pilarização propriamente dita, que se processa com a substituição dos cátions interlamelares das argilas do grupo das esmectitas, por oligômeros metálicos (Fig. 1). É possível produzir argilas pilarizadas pela intercalação tanto de cátions organometálicos complexos como de poli-hidroxicátions de $\mathrm{Al}$ [1-3], Fe [4, 5], $\mathrm{Zr} \mathrm{[6],} \mathrm{Cr}[4,5]$, Ti [7] e Ga [8, 9]. O material resultante da calcinação contém óxidos que funcionam como pilares, mantendo separadas as camadas e expondo as superfícies. Este processo tem como objetivo conferir microporosidade ao sistema, criando materiais contendo poros de dimensões complementares aos das zeólitas (maiores que 7 e menores que $20 \AA$ ); o processo é obtido simplesmente combinando uma esmectita com carga negativa baixa com um agente pilarizante catiônico de alta carga. Desta forma, se os agentes pilarizantes estiverem distribuídos de maneira homogênea sobre a superfície das lamelas; um sistema de canais bidimensional será criado [10-17].

Os parâmetros mais importantes que afetam a formação das propriedades destes polímeros são: concentração do íon metálico; grau de hidrólise; temperatura de preparação; tempo de envelhecimento e método de preparação $[12,14]$.

Atualmente é reconhecido que catalisadores homogêneos 


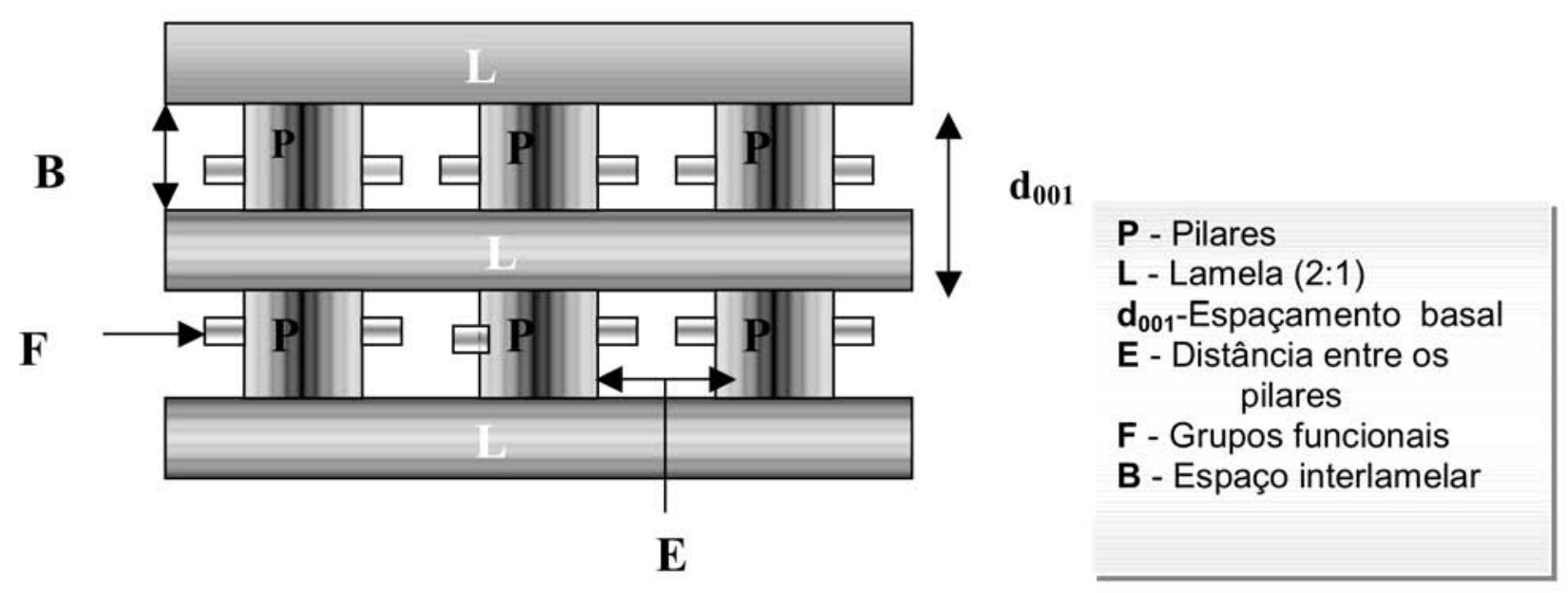

Figura 1: Representação esquemática do material produzido pelo processo de pilarização com o íon de titânio.

[Figure 1: Schematic representation of the material produced by pillaring process with titanium ion.]

empregados na alquilação de benzeno com 1-dodeceno, tais como o cloreto de alumínio e ácido fluorídrico, podem produzir a formação de produtos de ciclo alquilação como o 1,3-diaquil-indano. Estes compostos são considerados perigosos à saúde humana, podem trazer desequilíbrios ambientais, e afetam o valor agregado do produto final. Devido às desvantagens da catálise homogenea, foram propostos sólidos ácidos, com as pesquisas intensificadas devido a crise mundial do petróleo (1973) [1, 3]. Entre esses sólidos estão as zeólitas, argilas ativadas ácida e/ou termicamente e argilas pilarizadas com polioxicátions com valores altos de raio iônico.

A pilarização de esmectitas com titânio, foi efetuada pela primeira vez, com uma solução pilarizante de $\mathrm{TiCl}_{4} / \mathrm{HCl}$ [18]. O espaçamento basal, em temperatura de calcinação de $200{ }^{\circ} \mathrm{C}$, foi de $28 \AA$ [18]. Trabalhos posteriores realizados com a utilização de diferentes agentes pilarizantes, são descritos a seguir.

Óxido de titânio como agente pilarizante foi preparado pela hidrólise de tetraisopropilóxido de titânio seguido por peptização com $\mathrm{HCl}$. Os produtos resultantes apresentaram um espaçamento basal de $27 \AA$. O tamanho dos poros está diretamente relacionado com o tamanho das partículas, que são dependentes das condições de peptização [19].

Argilas pilarizadas pela intercalação de quatro alcóxidos de titânio diferentes. $\mathrm{O}$ agente pilarizante foi preparado pela adição de alcóxidos de titânio em uma solução de $\mathrm{HCl}$ sob constante agitação, variando a razão molar $\mathrm{HCl} /$ alcóxido. Foi investigada a estabilidade térmica e a distribuição dos pilares. O espaçamento basal obtido a temperatura ambiente foi de $14,9 \AA$ e a $600{ }^{\circ} \mathrm{C}$ foi de $10 \AA$ [20].

Mistura de uma solução de $\mathrm{TiCl}_{4}$ ou solução de etóxido de titânio com uma solução de glicerina e água. Obtiveram-se materiais com espaçamento basal de 21,3 $\mathrm{A}$ e área superficial de $379 \mathrm{~m}^{2} / \mathrm{g}$. A presença de glicerina foi essencial para obter materiais com grande estabilidade térmica [21].

O objetivo deste trabalho é a pilarização de uma esmectita do tipo beidelita, usando-se como agentes pilarizantes o
$\mathrm{TiCl}_{4}$ e o etóxido de titânio e a avaliação das propriedades estruturais e texturais das Ti-PILCs produzidas.

\section{MATERIAIS E MÉTODOS}

\section{Esmectita original}

A amostra natural de esmectita empregada pertence a um lajedo, situada no município de Serra Madurera no Estado do Acre. Com capacidade de troca catiônica (CTC) igual a $103 \mathrm{meq} / 100 \mathrm{~g}$ de argila, obtido por acetato de amônio $(2 \mathrm{~N}$, $\mathrm{pH}=8)$ e distância interbasal $\mathrm{d}(001)=15,30 \AA$. A amostra apresentou traços de caulinita e fração $\leq 2 \mu \mathrm{m}$ quando separada por sedimentação. A análise química desta argila apresenta os valores mostrados na Tabela I.

Tabela I - Análise química da argila natural. [Table I - Chemical analysis of the natural

\begin{tabular}{cc}
\hline Componentes & Teor $(\%)$ \\
\hline $\mathrm{SiO}_{2}$ & 28,66 \\
$\mathrm{Al}_{2} \mathrm{O}_{3}$ & 14,66 \\
$\mathrm{Fe}_{2} \mathrm{O}_{3}$ & 12,68 \\
$\mathrm{TiO}_{2}$ & 4,43 \\
$\mathrm{Na}_{2} \mathrm{O}$ & 0,79 \\
$\mathrm{MgO}$ & 1,08 \\
\hline
\end{tabular}

\section{Sínteses do polioxicátion de titânio}

O polioxicátion de Ti foi sintetizado por meio de dois métodos: A) o método do etóxido de titânio $\operatorname{Ti}\left(\mathrm{OC}_{2} \mathrm{H}_{5}\right)_{4}$ (2002) e B) método do $\mathrm{TiCl}_{4}$.

No método $\mathrm{A}$, o etóxido de titânio é tratado com uma solução de $\mathrm{HCl}$ a fim de se obter razão molar $\mathrm{HCl} / \mathrm{Ti}$ variando de 1 a 4 . Neste caso a solução é envelhecida a temperatura ambiente por $3 \mathrm{~h}$. A solução resultante é o polioxicátion de 
titânio. A intercalação da esmectita com polioxicátion de Ti neste trabalho foi efetuada sob as condições: razão molar $\left[\mathrm{H}^{+}\right] /\left[\mathrm{Ti}^{4+}\right]=1$ e Ti/argila $=25 \mathrm{mmolTi} / \mathrm{g}$ de argila. A mistura foi submetida à agitação mecânica em temperatura ambiente por $12 \mathrm{~h}$. O sólido resultante foi lavado com água deionizada até teste negativo para $\mathrm{Cl}^{-}$e depois seco ao ar. A pilarização foi feita a partir da calcinação do material intercalado em temperaturas de $300,450,600$ e $900{ }^{\circ} \mathrm{C}$.

No método B [24], a solução de titânio em concentração de 2,5 M foi preparada a partir de uma solução concentrada de $\mathrm{TiCl}_{4}$. Esta solução foi posta em contato com etanol por um período de $2 \mathrm{~h}$ em temperatura ambiente, e após este período, a solução resultante foi misturada com uma solução de glicerina e água (relação 2:1) em agitação mecânica mantida por $2 \mathrm{~h}$. O tempo de envelhecimento adotado foi de $24 \mathrm{~h}$.

\section{Processo de pilarização}

As matrizes em forma natural foram preparadas previamente em atmosfera de etilenoglicol, em temperatura ambiente por um período de $24 \mathrm{~h}$. Posteriormente foram colocadas em agitação magnética com as soluções pilarizantes, por um período de $3 \mathrm{~h}$ a $25^{\circ} \mathrm{C}$ (processo de intercalação). Após a intercalação, as amostras foram filtradas, lavadas, secas a $60^{\circ} \mathrm{C}$ e calcinadas em temperaturas de $300,450,600$ e $700^{\circ} \mathrm{C}$, e conservadas em dessecador pelo período de utilização dos métodos de caracterização.

\section{Métodos de caracterização}

Os materiais pilarizados foram submetidos aos seguintes ensaios de caracterização:

Difração de raios $X$ : O equipamento utilizado foi Philips PW1050, operando com radiação $\operatorname{CuK}_{\alpha}(1,54060 \AA$ ), a $45 \mathrm{kV}$ e $35 \mathrm{~mA}$, com passo de $\theta=1 \%$ min. Foi empregado o método do pó sem orientação preferencial.

Análise química: A análise química das amostras (natural e pilarizada) foi realizada pelo método de absorção atômica para os elementos $\mathrm{Na}, \mathrm{K}, \mathrm{Ca}, \mathrm{Mg}$ e $\mathrm{Al}$; para os elementos $\mathrm{Si}$, Fe e Ti foi utilizado o método de espectroscopia na região do visível (Fe sob a forma do complexo ortofenantrolina ferrosa e Ti como peróxido complexo).

Infravermelho: Para a obtenção dos espectros FTIR foram utilizados pastilhas prensadas a vácuo contendo $0,200 \mathrm{~g}$ de $\mathrm{KBr}$ e $0,0013 \mathrm{~g}$ de amostra pulverizada e um espectrômetro de absorção molecular na região IV com transformada de Fourier, Perkin Elmer modelo FT-IR 1760 X.

Análises térmicas: Para a obtenção das curvas de análises térmicas (ATD/ATG) foi utilizado um termoanalisador Stanton Redcroft. A análise foi feita em uma faixa de temperatura de $25^{\circ} \mathrm{C}$ a $1100{ }^{\circ} \mathrm{C} \mathrm{com} \mathrm{uma} \mathrm{taxa} \mathrm{de} \mathrm{aquecimento} 20^{\circ} \mathrm{C} / \mathrm{min}$.

Análise Textural: As determinações da área específica (AE) pelo método BET (Brunauer-Emmett-Teller), do volume total dos poros (VTP) pelo método BJH (BarretJoyner-Halenda) e do diâmetro médio dos poros (DMP), foram feitas a partir das isotermas de adsorção-dessorção, obtidas em um analisador de adsorção gasosa Quantachrome/ Nova 1200/5. A quantidade de cada amostra utilizada foi $0,249 \mathrm{~g}$, aquecida previamente a $150{ }^{\circ} \mathrm{C}$ por $1 \mathrm{~h}$.

MEV e microanálise: Para os estudos de microscopia eletrônica de varredura e espectroscopia de raios $\mathrm{X}$ por energia dispersiva, as amostras foram preparadas em laminas orientadas a $25^{\circ} \mathrm{C}$ e metalizadas com ouro. O microscópio utilizado foi LEO- Zeiss, $430 \mathrm{Vp}$.

\section{RESULTADOS E DISCUSSÃO}

\section{Difração de raios $X$}

Na Fig. 2 estão apresentados os resultados da matriz esmectítica na forma natural e pilarizada nas temperaturas de $300,450,600$ e $700{ }^{\circ} \mathrm{C}$. Observou-se um comportamento dos valores de $\mathrm{d}_{001}$ bastante semelhante nas esmectitas pilarizadas pelos dois métodos abordados neste trabalho. Com o aumento da temperatura de calcinação, o valor de

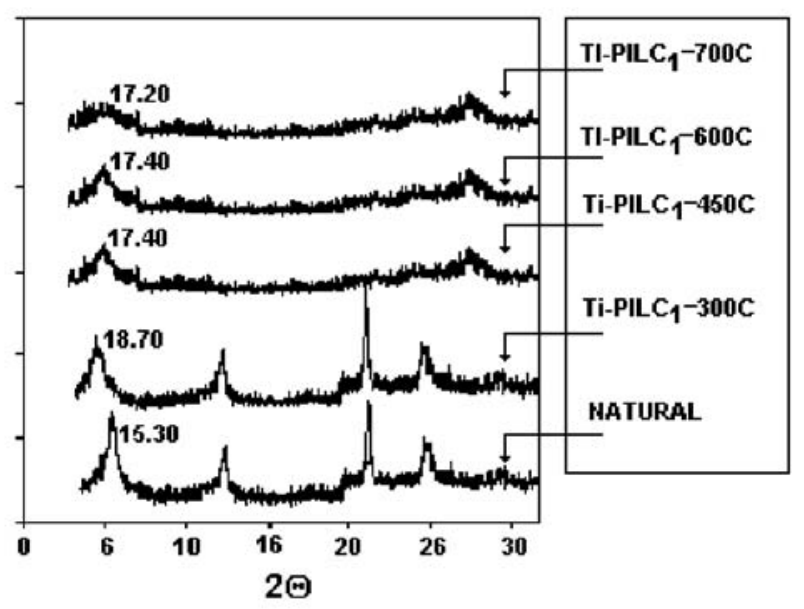

\section{(A) MÉTOdo A}

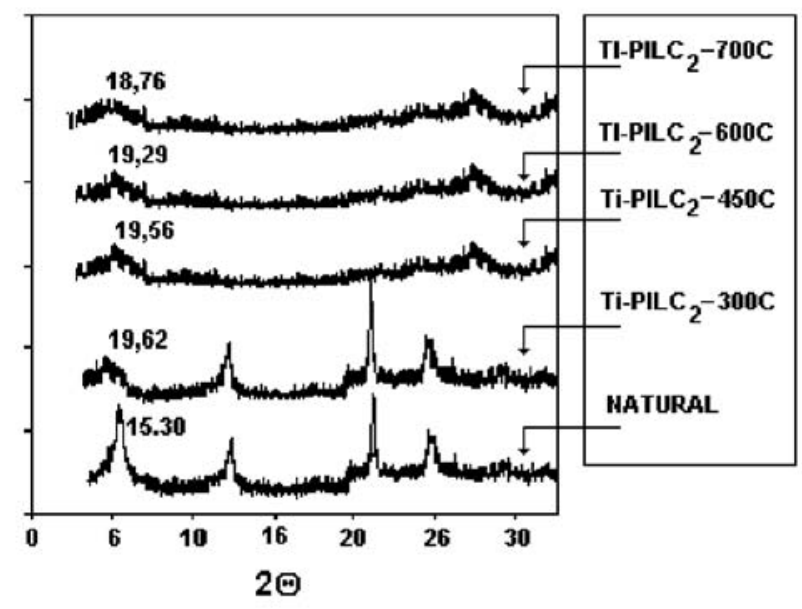

\section{(B) MÉTODO B}

Figura 2: Difratogramas de raios $\mathrm{X}$ das matrizes pilarizadas com titânio pelos métodos em estudo.

[Figure 2: X-ray diffraction patterns of the pillared matrix with titanium.] 
$\mathrm{d}_{001}$ diminui, chegando este pico ao total desaparecimento acima de $700{ }^{\circ} \mathrm{C}$. Vários fatores podem ter interferido neste resultado, sendo um dos mais relevantes a maior produção do íon titanato em solução; uma outra hipótese seria a posição do íon intercalante dentro da região interlamelar, que pode ter interferido na ligação pilar-lâmina e consequentemente na formação de um pseudo pilar. Com a calcinação a baixa temperatura, este pilar sofreria desidroxilação significativa, ocorrendo uma pilarização parcial.

$\mathrm{O}$ método A produziu esmectita pilarizada com valores menores de $\mathrm{d}_{001}$ que o método B. Entretanto foi observado que a cristalinidade da matriz quando pilarizada pelo método A, foi menos comprometida do que a matriz pilarizada pelo método $\mathrm{B}$. O método A produziu um material de maior grau de cristalinidade, observado pela formação do pico referente ao plano $\mathrm{d}_{001}$, permanecendo esta cristalinidade em temperaturas de calcinação mais elevadas.

Na Fig. 3 está apresentado o comportamento do valor de $d_{001}$ frente a variação de temperatura de calcinação. Observa-se que o valor de $d_{001}$ decresce com o aumento da temperatura, fato que pode ser explicado pela ocorrência da desidroxilação do íon intercalante, à medida que se transforma em pilar (óxido). O decréscimo do valor de $\mathrm{d}_{001}$ é promovido pela diminuição do tamanho do íon intercalante, portanto, haverá aproximação entre as lamelas. É observado que na pilarização pelo método $\mathrm{A}$ (Ti-PILC ${ }_{1}$ ), há uma estabilização deste valor na faixa de temperatura entre 450 e $600^{\circ} \mathrm{C}$, fato que não acontece no comportamento da matriz, quando pilarizada pelo método B (Ti-PILC 2 ). Nas duas matrizes é evidenciado que, a partir da temperatura de $600{ }^{\circ} \mathrm{C}$, o comportamento de desidroxilação é o mesmo. Observa-se que diminuição mais importante se dá na matriz Ti-PILC ${ }_{1}$ na faixa de temperatura entre 300 e $450{ }^{\circ} \mathrm{C}$. Este resultado é semelhante ao reportado em 2002 [21].

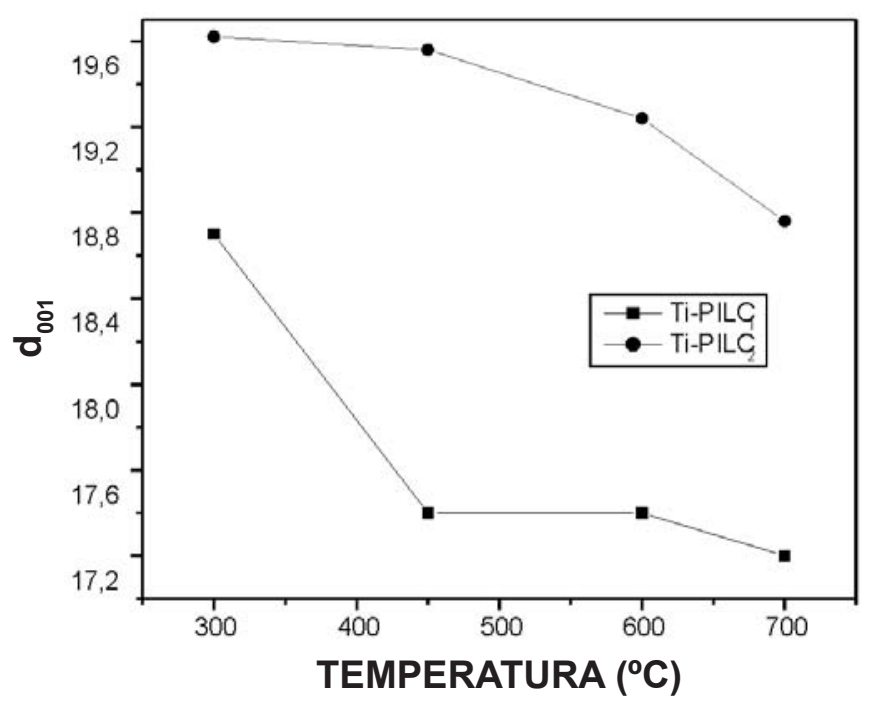

Figura 3: Comportamento de $\mathrm{d}_{001}$ com a temperatura de calcinação das duas matrizes pilarizadas com Ti.

[Figure 3: $d_{001}$ behavior with the calcination temperature of the two pillared matrix with Ti.]
Espectroscopia no infravermelho com transformada de Fourier (FTIR)

\section{Análise das soluções pilarizantes}

Por meio do espectro de FTIR foi possível observar, analisando as soluções pilarizantes, uma banda de absorção na região de $547 \mathrm{~cm}^{-1}$ na solução obtida pelo método Ae banda em $576 \mathrm{~cm}^{-1}$ na solução obtida pelo método B. Estas bandas podem ser atribuídas à ligação Ti-O em solução, obtidas a partir $\mathrm{Ti}\left(\mathrm{OC}_{2} \mathrm{H}_{5}\right)_{4} / \mathrm{HCl}$ e TiCl 4 /Etanol (Fig. 4). Outras bandas observadas em regiões de maiores intensidades, podem ser atribuídas às ligações de $\mathrm{C}-\mathrm{H}$ e $\mathrm{O}-\mathrm{H}$.

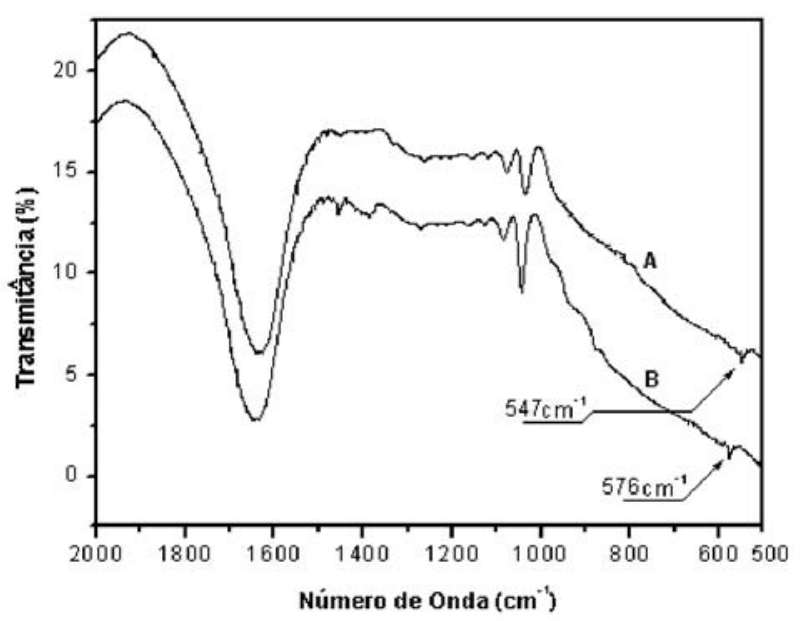

Figura 4: Espectros de FTIR da solução pilarizante preparada com $\mathrm{Ti}\left(\mathrm{OC}_{2} \mathrm{H}_{5}\right)_{4}$.

[Figure 4: FTIR spectra of the pillaring solution prepared with $\left.\mathrm{Ti}\left(\mathrm{OC}_{2} \mathrm{H}_{5}\right)_{4} \cdot\right]$

\section{Análise das argilas pilarizadas}

Através das análises dos espectros de FTIR, não foi observado a pequena banda de absorção na região de $576 \mathrm{~cm}^{-1}$ na esmectitas pilarizadas, como aquela observada nas análises das soluções pilarizantes. A hipótese mais provável é a ocorrência da sobreposição entre o pico referente a ligação Ti-O e os picos das ligações Si-O $\left(693-463 \mathrm{~cm}^{-1}\right)$, porém, é possível observar banda na região de $1186 \mathrm{~cm}^{-1}$. Esta banda é apenas observada nos espectros das esmectitas pilarizadas, que pode representar ligações de hidrogênio na estrutura da esmectita, podendo este fato ter relação com a formação de novos sítios ácidos no interior da região interlamelar (ligação pilar-lamela) e também formação de ligações na parte externa dos pilares (Fig. 5).

\section{Análise textural}

Na Tabela II estão apresentados os resultados da análise textural: área específica, volume total de poros e diâmetro médio dos poros. Foi verificado que com a pilarização o valor da área específica aumentou, atingindo o valor máximo na 


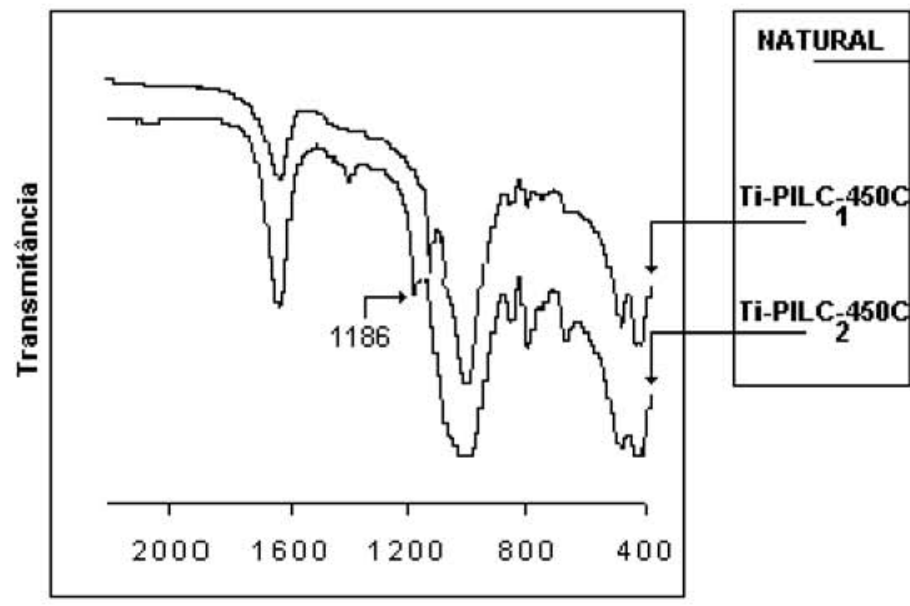

Húmero de onda $\left(\mathrm{cm}^{-1}\right)$

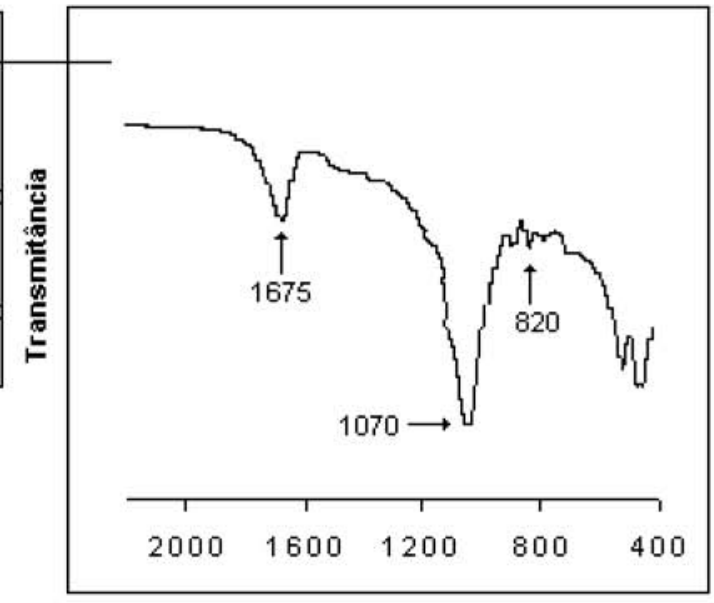

Húmero de onda $\left(\mathrm{cm}^{-1}\right)$

Figura 5: Espectros FTIR das matrizes natural e pilarizadas.

[Figure 5: FTIR specta of the natural and pillared matrix.]

matriz pilarizada pelo método A (Ti-PILC $\left.{ }_{1}-450\right)$. Analisando as isotermas de adsorção/dessorção de $\mathrm{N}_{2}$, formadas para as amostras pilarizadas, é observado que elas pertencem à classificação do tipo IV, segundo BDDT (Brunauer, Deming, Deming e Teller) [20, 22, 23] (Fig. 6). Houve a formação de ciclo de histerese em altas pressões relativas, nas duas matrizes pilarizadas, característica dos materiais mesoporosos, podendo conter microporos. Um ligeiro afastamento da curva é observado a baixas pressões. Este tipo de efeito está relacionado com fenômeno de expansão, que é característico de materiais cujo grau de expansão do sólido, durante a adsorção, depende da rigidez deste sólido.

A distribuição do volume de poros está apresentada na Fig. 7 (valores encontrados pelo método BJH) [3]. A curva de distribuição tem um comportamento unimodal para as argilas pilarizadas, com picos em torno de $30 \AA$ A. A distribuição do volume de poros obtidos com a argila natural apresentou comportamento bimodal com picos em 10 e $12 \AA$ ( Fig. 7)

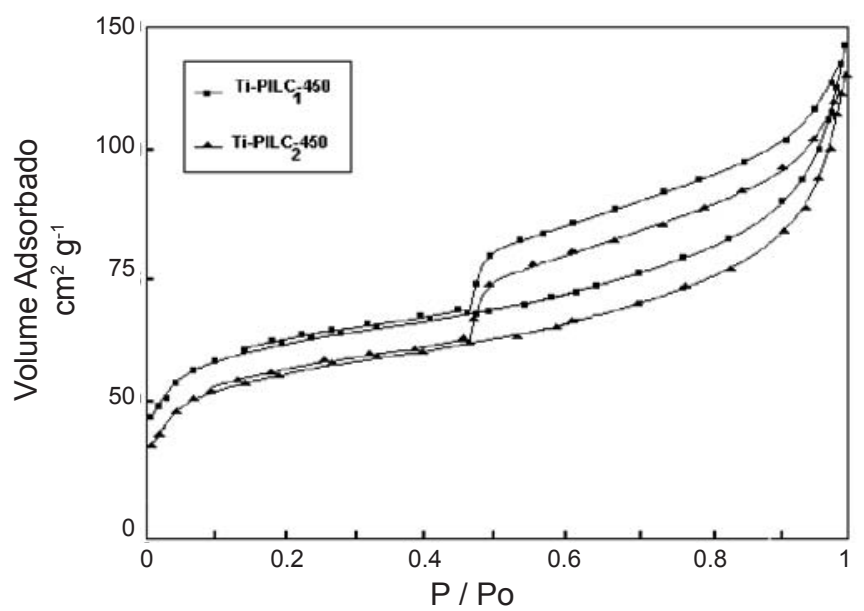

Figura 6: Isortermas de adsorção-dessorção obtidas com $\mathrm{N}_{2}$ das argilas pilarizadas.

[Figure 6: Adsorption-desorption isotherms obtained with $\mathrm{N}_{2}$ of the pillared clay.]

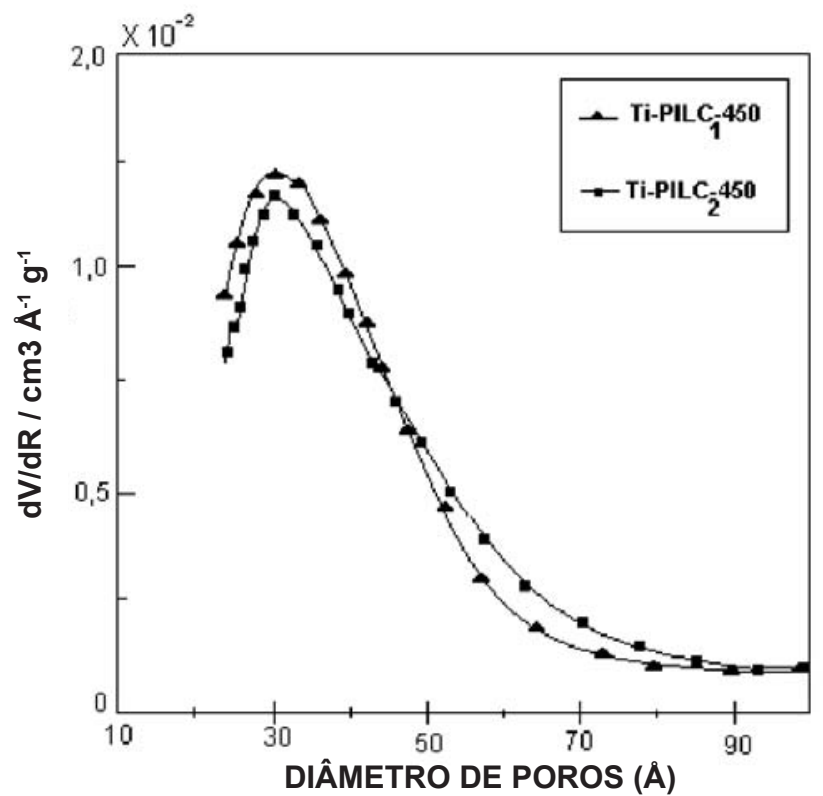

Figura 7: Curvas de distribuição de tamanhos de poros das argilas pilarizadas.

[Figure 7: Poros size distribution curves of the pillared clay.]

Tabela II - Resultados da análise textural das esmectitas natural e pilarizadas.

[Table II - Results of the textural analyses of pillared and natural smectites.]

\begin{tabular}{cccc}
\hline Amostras & $\begin{array}{c}\text { AE } \\
\left(\mathrm{m}^{2} / \mathrm{g}\right)\end{array}$ & $\begin{array}{c}\text { VTP } \\
\left(\mathrm{cm}^{3} / \mathrm{g}\right)\end{array}$ & $\begin{array}{c}\text { DMP } \\
(\mathrm{nm})\end{array}$ \\
\hline Natural & 44,37 & 0,12 & 4,509 \\
Ti-PILC $_{1}-450$ & 188,72 & 0,23 & 7,530 \\
Ti-PILC $_{2}-450$ & 171,80 & 0,27 & 7,762 \\
\hline
\end{tabular}




\section{Análise térmica}

Através das curvas de análise térmica (Figs. 8 e 9) foi verificado que a perda de água é maior na matriz pilarizada com o método de síntese B. Este resultado pode ser causado pela existência de maior número de pilares na região interlamelar. Observa-se também uma perda maior de água na amostra Ti-PILC ${ }_{2}-450$, em temperatura acima de $500{ }^{\circ} \mathrm{C}$, com sinal de desidroxilação considerável. Esta desidroxilação ocorre em temperatura mais alta que a desidroxilação da matriz Ti-PILC ${ }_{1}-450$, comprovando maior resistência a temperaturas mais altas. Na temperatura de aproximadamente $200{ }^{\circ} \mathrm{C}$ há uma reação exotérmica na matriz Ti-PILC $\mathrm{C}_{2}-450$. Esta reação pode ser atribuída a desidroxilação prematura de pilares mal formados ou mal posicionados, dentro da região interlamelar, como por exemplo, pilares concentrados nas bordas desta região, que pode ser confirmado pela análise de difração de raios $\mathrm{X}$, quando verificado a forma da curva $\mathrm{d}_{001}$.

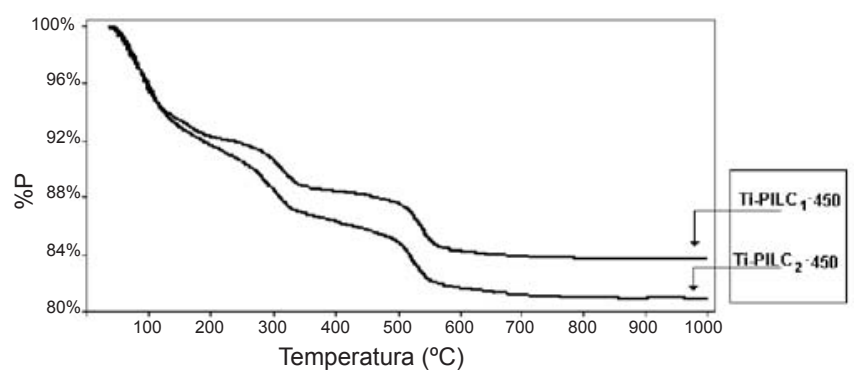

Figura 8: Curvas de ATG das matrizes pilarizadas a $450^{\circ} \mathrm{C}$.

[Figure 8: TGA curves of the pillared matrix at $450^{\circ} \mathrm{C}$.]

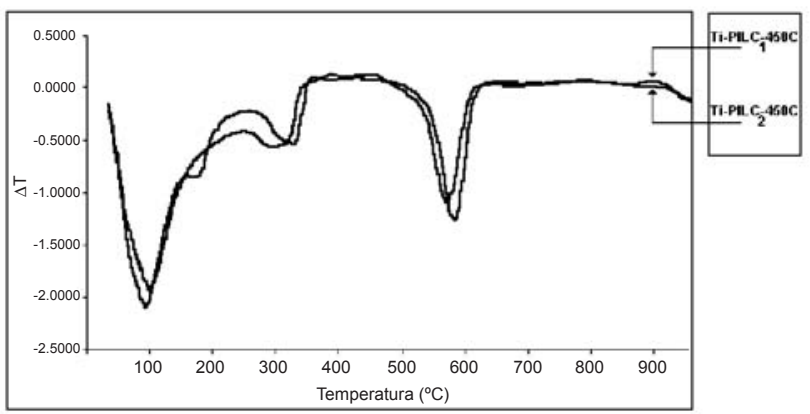

Figura 9: Curvas de ATD das matrizes pilarizadas a $450^{\circ} \mathrm{C}$. [Figure 9: DTA curves of the pillared matrix at $450^{\circ} \mathrm{C}$.]

\section{Microscopia eletrônica de varredura}

Com base nos resultados do MEV (Fig. 10) fornecidos para as matrizes pilarizadas com os métodos em estudo, é possível afirmar que o processo de pilarização não produz alterações visíveis na morfologia da esmectita, quando pilarizada com titânio. Nos dois métodos pesquisados foi observado que em todos os experimentos a esmectita permaneceu na forma original, apresentando morfologia de forma foliada com ilita nas extremidades, fato comprovado pela análise elementar pontual (EDS).

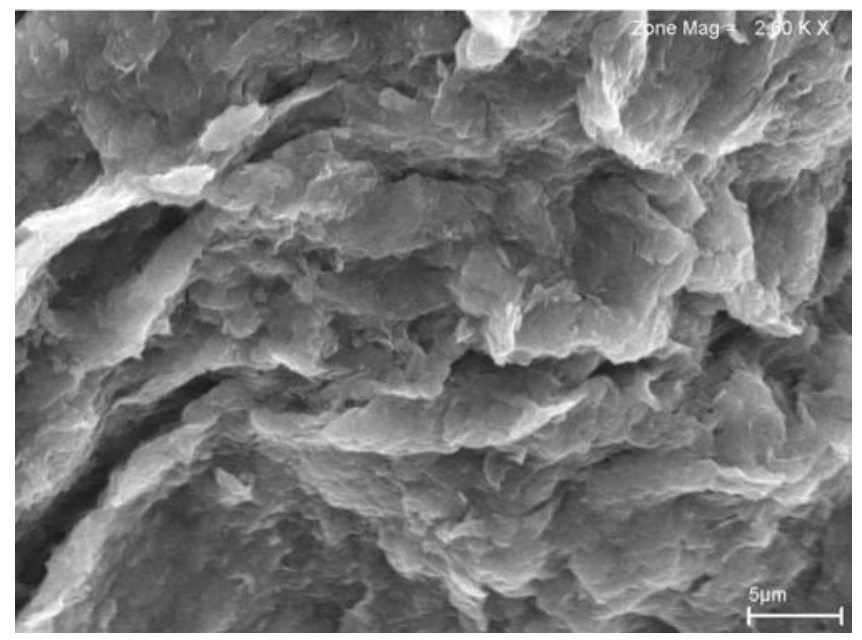

(A) Natural

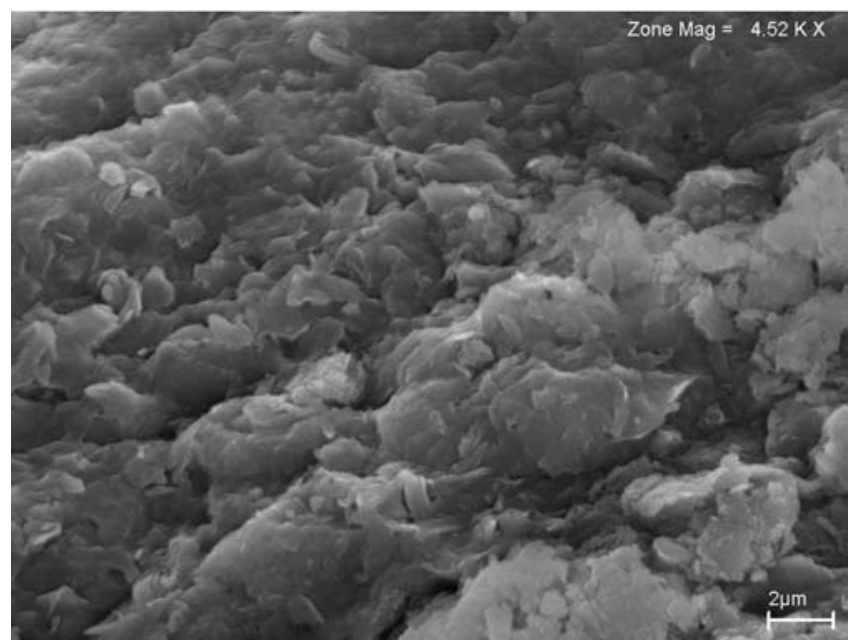

(B) Pilarizada (Ti-PILC $1-450)$

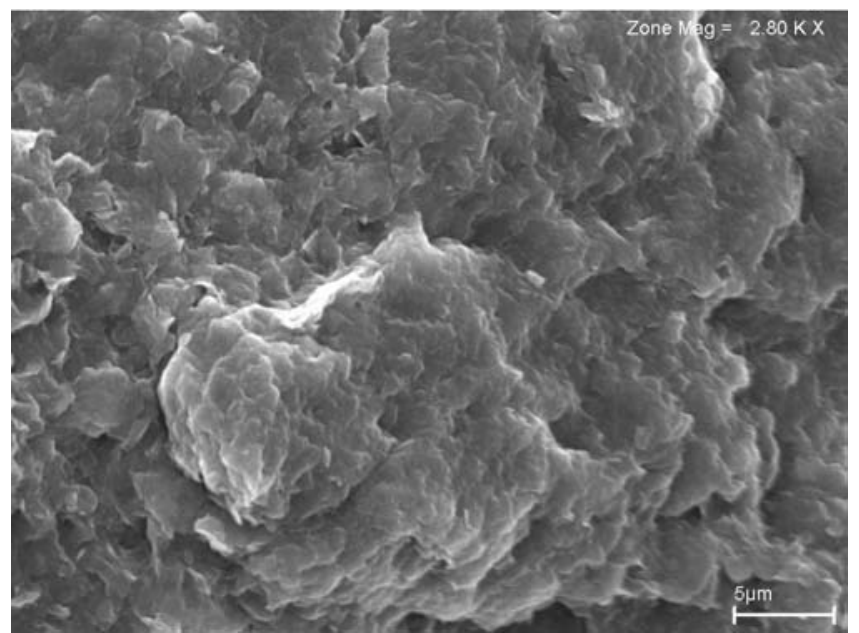

(C) Pilarizada (Ti-PILC 2 -450)

Figura 10: Micrografias obtida por microscopia eletrônica de varredura das matrizes natural e pilarizadas.

[Figure 10: Scanning electron microscopy micrographs of the natural and pillared matrix.] 


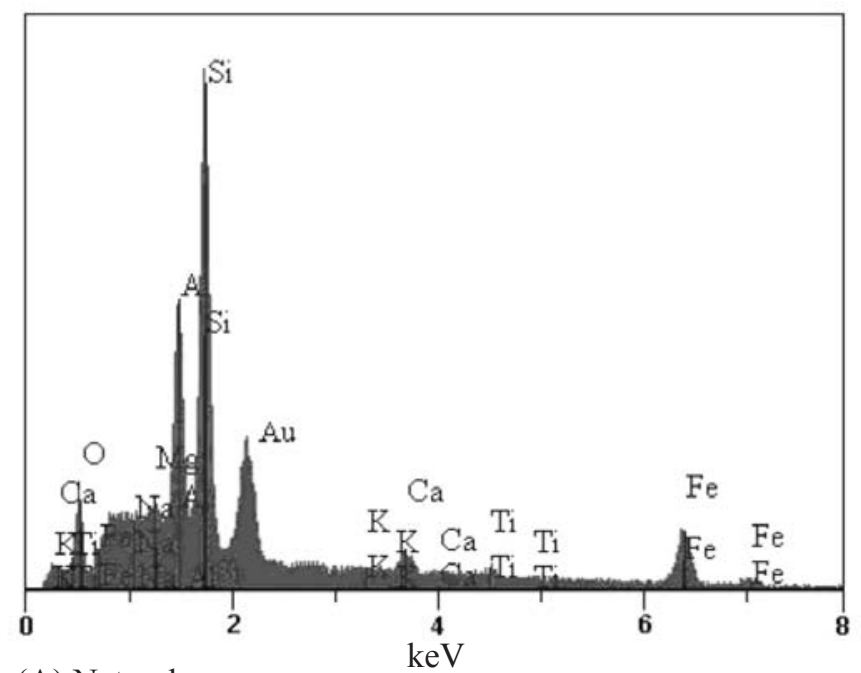

(A) Natural

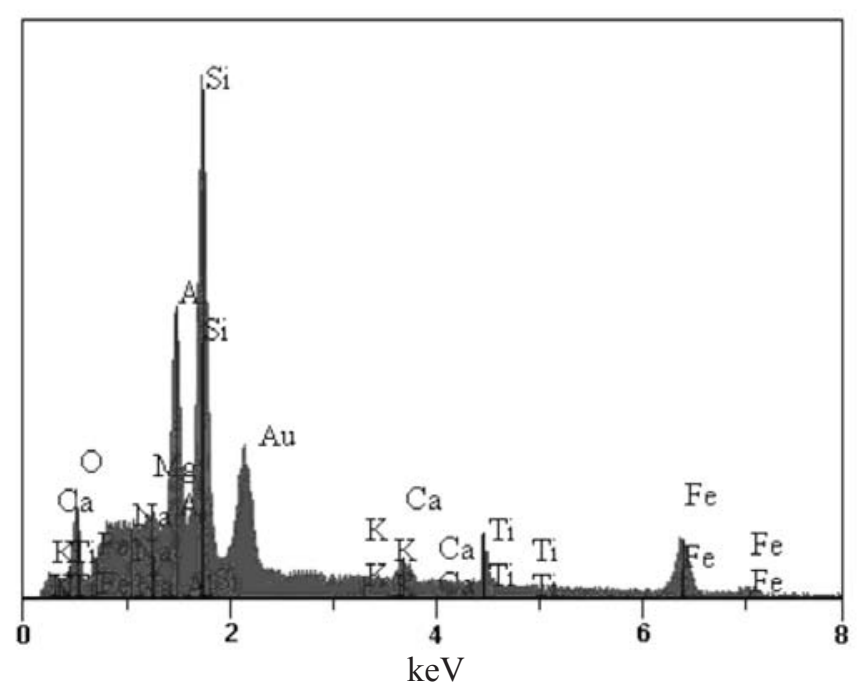

(B) Pilarizada (Ti-PILC 1 -450)

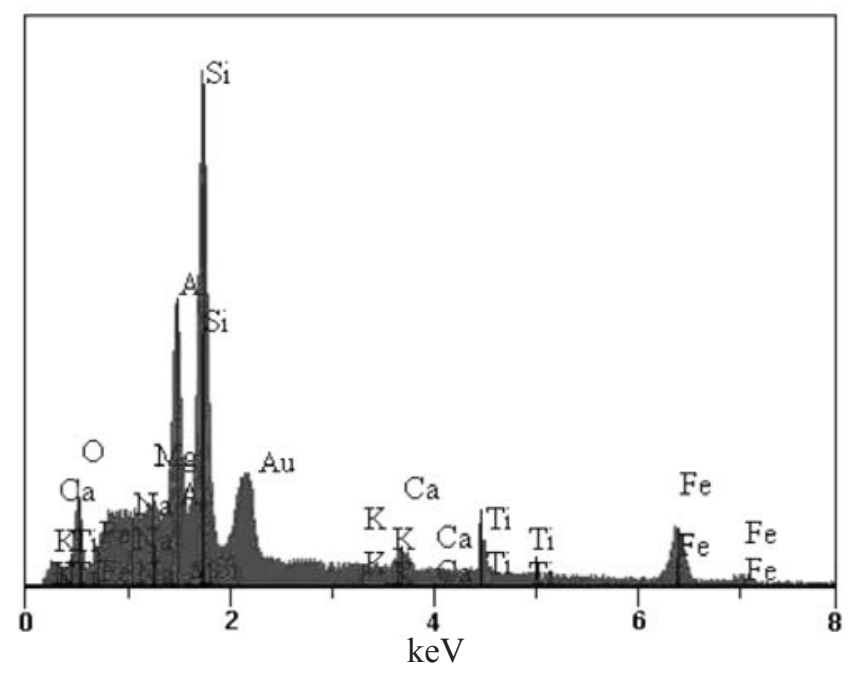

(C) Pilarizada (Ti-PILC $2-450)$

Figura 11: Espectros de EDS das matrizes natural e pilarizadas. [Figure 11: EDS results of the natural and pillared matrix.]

\section{CONCLUSÕES}

A matriz pilarizada (Ti-PILC1-450), produzida pelo método $\mathrm{A}$, obteve maior conservação no grau de cristalinidade do que a matriz pilarizada pelo método $\mathrm{B}$, onde se observam picos mais largos, indicando baixa cristalinidade. Possivelmente isto foi provocado por uma distribuição irregular de pilares na região interlamelar, que podem ter maior concentração nas bordas deixando a região central com baixa densidade de pilares, pelo colapso parcial da estrutura.

As analises de FTIR mostraram uma pequena banda na região de $576 \mathrm{~cm}^{-1}$, atribuída à ligação O-Ti dentro da região interlamelar e uma banda na região próxima a 1200 $\mathrm{cm}^{-1}$, atribuída às ligações de hidrogênio; esta banda pode ser a comprovação da formação de novos sítios ácidos na estrutura esmectítica relacionada à presença de pilares e consequentemente a seus grupos funcionais (M-O) [18].

As análises térmicas (ATD/ATG) mostraram que a matriz Ti-PILC ${ }_{2}-450$ teve maior hidratação com o processo de pilarização do que a matriz Ti-PILC 1 -450; apresentam perda de água maior em todas as reações exotérmicas no intervalo estudado, devido provavelmente ao maior numero de grupos $\mathrm{OH}$ presos as superfícies internas da matriz e ancorados na parte externa do pilar, estas hidroxilas são responsáveis pelo fenômeno chamado de inchamento da estrutura.

Analisando as micrografias obtidas por microscopia eletrônica de varredura, constatou-se a conservação na morfologia das partículas, levando a conclusão de que o processo de pilarização não interfere na forma original das partículas da matriz.

O estudo concluiu que o método A [21] obteve maior eficiência, produzindoumaesmectita pilarizadatermicamente mais estável e com melhores propriedades texturais.

\section{AGRADECIMENTOS}

Ao Centro de Geociências (UFPA) com o seu corpo técnico, ao IQ-UNICAMP e ao $\mathrm{CNPq}$ pelo suporte financeiro.

\section{REFERÊNCIAS}

[1] S. Moreno, E. Guitierrez, A. Alvarez, N. G. Papayannakos, G. Poncelet, Appl. Catal. 165 (1997) 103-104.

[2] M. L. Occelli, R. M. Tindwa, Clay Clay Min. 31 (1983) 22-28.

[3] L. Storaro, M. Lenarda, A. Rinaldi, Microporous Mesoporous Mater. 6 (1996) 5-63.

[4] P. Cañizares, J. L. Valverde, M. R. Sun Kou, C. B. Molina, Microporous Mesoporous Mater. 29 (1999) 267-281.

[5] N. Maes, I. Hielen, P. Cool, P. E. F. Vansant, Appl. Clay Sci. 12 (1997) 43-60.

[6] M. R. Sun Kou, S. Mendioroz, M. L. Guijarro, Termochim. Acta 323 (1998) 145-157.

[7] M. Sychev, M. Rozwadowiski, A. P. B. Sommen, V. H. J. de Beer, R. A. van Santen, Microporous Mesoporous Mater. 
37 (2000) 187-200.

[8] S. M. Bradley, R. A. Kidd, J. Catal. 141 (1993) 239249.

[9] K. B. Brandt, R. A. Kidd, Appl. Catal. 165 (1997) 327333.

[10] G. W. Blindley, R. E. Sempels, Clay Clay Min. 12 (1997) 229-237.

[11] T. J. Pinnavaia, Science, 220 (1983) 365-371.

[12] T. J. Pinnavaia, Nature, 309 (1984) 604-607.

[13] W. Jones, Catal. Today 2 (1998).357-367.

[14] D. Zhao, Chem. Mater. 4 (1993)1433-1437.

[15] B. C. Perguer, A. Corma, V. Fornés, Química Nova 22, 5 (1999) 693-709.

[16] B. C. Perguer, A. Corma, V. Fornés, Química Nova, 22, 5 (1999) 649-653.
[17] D. Plee, L. Gatineau, J. J. Fripiat, Clay Clay Min. 35 (1997) 81-88.

[18] J. Sterte, Clay Clay Min. 35 (1986) 658-664.

[19] S. Yamanaka, T. Nishihara, M. Hattori, Mater. Res. Soc. (1988).

[20] H. L. del Castillo, A. Gil, P. Grange, Clay Clay Min. 44, 5 (1996) 706-709.

[21] J. L. Valverde, P. Sanchez, F. Dourado, C. B. Molina, A. Romero, Microporous Mesoporous Mater. 54 (2002) 155-165. [22] A. Bernier, L. F. Admaiai, P. Grange, Appl. Catal. 77 (1991) 269-281.

[23] C. Ooka, A. Yoshide, M. Horio, K. Suzuki, T. Hattori, Appl. Cat. 41 (2003) 313-321.

[24] J. T. Lin, S. J. Jong, S. Cheng, Microp. Mater. 1 (1993) 287.

(Rec. 23/10/2005, Rev. 28/02/2006, Ac. 16/05/2006) 